\title{
Box model of migration in channels of migration networks
}

\author{
Nikolay K. Vitanov, Kaloyan N. Vitanov, Tsvetelina Ivanova \\ Institute of Mechanics, Bulgarian Academy of Sciences, Acad. \\ G. Bonchev Str., Bl. 4, 1113 Sofia, Bulgaria
}

\begin{abstract}
We discuss a box model of migration in channels of networks with possible application for modelling motion of migrants in migration networks. The channel consists of nodes of the network (nodes may be considered as boxes representing countries) and edges that connect these nodes and represent possible ways for motion of migrants. The nodes of the migration channel have different "leakage", i.e. the probability of change of the status of a migrant (from migrant to nonmigrant) may be different in the different countries along the channel. In addition the nodes far from the entry node of the channel may be more attractive for migrants in comparison to the nodes around the entry node of the channel. We discuss below channels containing infinite number of nodes. Two regimes of functioning of these channels are studied: stationary regime and non-stationary regime. In the stationary regime of the functioning of the channel the distribution of migrants in the countries of the channel is described by a distribution that contains as particular case the Waring distribution. In the non-stationary regime of functioning of the channel one observes exponential increase or exponential decrease of the number of migrants in the countries of the channel. It depends on the situation in the entry country of the channel for which scenario will be realized. Despite the non-stationary regime of the functioning of the channel the asymptotic distribution of the migrants in the nodes of the channel is stationary. From the point of view of the characteristic features of the migrants we discuss the cases of (i) migrants having the same characteristics and (ii) two classes of migrants that have differences in some characteristic (e.g., different religions).
\end{abstract}




\section{Introduction}

Flows in complex networks are important for existence and functioning of the systems containing such networks. Human migration (the permanent or semipermanent change of residence that involves e.g., the relocation of individuals, households or moving groups between geographical locations [19]) is one example of such a flow [11, [13. - Large external migration flows reached Europe in the last years and this makes the study of migration very actual topic. In addition the internal migration studies are important for taking decisions about economic development of regions of a country [1]-4], [10, [12], [14, 25], 26], 43]. Examples for results from such studies is e.g., the Hecksehr-Ohlin theorem for economic use of a country relative abundant factors such as labor as well as the factor-price equalization theorem [2]. Migrant flows may be modelled by deterministic or stochastic tools [15], [18, [20, 21], 22, 44] and the corresponding migration models can be classified as probability models [45], [46] or deterministic models with respect to their mathematical features. Examples for probability models are the exponential model, multinomial model or Markov chain models of migration. One of the most famous deterministic models of migration is the gravity model of migration [12. The gravity model may be extended in different ways, e.g., to include the income and unemployment in the two regions.

Human migration is closely connected to ideological struggles [32, 37 and waves and statistical distributions in population systems [39], [28], 30, 31, [40. In this article we shall consider a box model of a flow of migrants in a channel (sequence of countries) of a migration network. The nodes (countries) will be considered as boxes (cells) where the following processes happen: inflow and outflow of migrants and "leakage" (change of the status of migrant). Migrants enter the channel from the entry country and move through the channel. The different nodes of the channel (the different countries) are assumed to have different rate of "leakage" (i.e. different probabilities of change of the status of a person form the migrant to non-migrant).

The paper is organized as follows. In Sect.2 the model for moving of substance in a channel containing an infinite number of nodes is discussed. Two regimes of functioning of the channel: stationary regime (the amount of the substance in the entry box of the channel doesn't change) and non-stationary regime (amount of substance in the entry box of the channel decreases or increases exponentially) are described. Statistical distributions of the amount of substance in the nodes of the channel are obtained. A particular case of the distribution for the stationary regime of functioning of the channel is the 
Waring distribution. Sect. 3 is devoted to the case of two immiscible substances moving through the channel channel. In Sect. 4 we relate the obtained mathematical results to the movement of migrants through a migration channel.

\section{Channel containing infinite number of nodes}

Inspired by the models in [23], 27], and [29] we consider a model of moving of a substance through a channel as follows. The channel contains infinite number of nodes and each node can be considered as a cell. The cells are indexed in succession by non-negative integers. The first cell has index 0 . We assume that an amount $x$ of some substance is distributed among the cells and this substance can move from one cell to another cell. Let $x_{i}$ be the amount of the substance in the $i$-th cell. Then

$$
x=\sum_{i=0}^{\infty} x_{i}
$$

The fractions $y_{i}=x_{i} / x$ can be considered as probability values of distribution of a discrete random variable $\zeta$

$$
y_{i}=p(\zeta=i), i=0,1, \ldots
$$

The content $x_{i}$ of any cell can change because of the following 3 processes:

1. Some amount $s$ of the substance $x$ enters the channel from the external environment through the 0 -th cell;

2. Rate $f_{i}$ from $x_{i}$ is transferred from the $i$-th cell into the $i+1$-th cell;

3. Rate $g_{i}$ from $x_{i}$ leaks out the $i$-th cell into the external environment.

The above processes can be modeled mathematically by the system of ordinary differential equations:

$$
\begin{aligned}
& \frac{d x_{0}}{d t}=s-f_{0}-g_{0} \\
& \frac{d x_{i}}{d t}=f_{i-1}-f_{i}-g_{i}, i=1,2, \ldots
\end{aligned}
$$

The following forms of the amount of the moving substances may be assumed $\left(\alpha, \beta, \gamma_{i}, \sigma\right.$ are constants)

$s=\sigma x_{0} ; \sigma>0$ 
$f_{i}=(\alpha+\beta i) x_{i} ; \quad \alpha>0, \beta \geq 0 \rightarrow$ cumulative advantage of higher cells

$g_{i}=\gamma_{i} x_{i} ; \quad \gamma_{i} \geq 0 \rightarrow$ non-uniform leakage over the cells

The rules (4) differ from the rules in [23] as follows:

1. $s$ is proportional to the the amount of the substance $x_{0}$ in the 0 -th node. In [23] $s$ is proportional to the amount $x$ of the substance in the entire channel;

2. Leakage rates $\gamma_{i}$ are different for the different nodes. In [23] and [29] the leakage rate is constant and equal to $\gamma$ for all nodes of the channel (i.e., there is uniform leakage over the cells).

Substitution of Eqs.(4) in Eqs.(3) leads to the relationships

$$
\begin{aligned}
\frac{d x_{0}}{d t} & =\sigma x_{0}-\alpha x_{0}-\gamma_{0} x_{0} \\
\frac{d x_{i}}{d t} & =[\alpha+\beta(i-1)] x_{i-1}-\left(\alpha+\beta i+\gamma_{i}\right) x_{i} ; \quad i=1,2, \ldots
\end{aligned}
$$

There are two regimes of functioning of the channel and realization of one of them depends on the situation in the 0-th node (the entry cell). The regimes are stationary regime and non-stationary regime.

\subsection{Stationary regime of functioning of the chan- nel}

In the stationary regime of the functioning of the channel $\sigma=\alpha+\gamma_{0}$ which means that $x_{0}$ (the amount of the substance in the 0 -th cell of the channel) is free parameter. In this case the solution of Eqs.(5) is

$$
x_{i}=x_{i}^{*}+\sum_{j=0}^{i} b_{i j} \exp \left[-\left(\alpha+\beta j+\gamma_{j}\right) t\right]
$$

where $x_{i}^{*}$ is the stationary part of the solution. For $x_{i}^{*}$ one obtains the relationship

$$
x_{i}^{*}=\frac{\alpha+\beta(i-1)}{\alpha+\beta i+\gamma_{i}} x_{i-1}^{*}
$$

The corresponding relationships for the coefficients $b_{i j}$ are

$$
b_{i j}=\frac{\alpha+\beta(i-1)}{\gamma_{i}-\gamma_{j}+\beta(i-j)} b_{i-1, j}, j=0,1, \ldots, i-1
$$

From Eq.(7) one obtains

$$
x_{i}^{*}=\frac{[k+(i-1)] !}{(k-1) ! \prod_{j=1}^{i}\left(k+j+a_{j}\right)} x_{0}^{*}
$$


where $k=\alpha / \beta$ and $a_{j}=\gamma_{j} / \beta$. The form of the corresponding stationary distribution $y_{i}^{*}=x_{i}^{*} / x^{*}$ (where $x^{*}$ is the amount of the substance in all of the cells of the channel) is

$$
y_{i}^{*}=\frac{[k+(i-1)] !}{(k-1) ! \prod_{j=1}^{i}\left(k+j+a_{j}\right)} y_{0}^{*}
$$

Let us consider the particular case where $a_{0}=a_{1}=\ldots=a$. In this case the distribution from Eq.(10) is reduced to the distribution:

$$
P(\zeta=i)=P(\zeta=0) \frac{(k-1)^{[i]}}{(a+k)^{[i]}} ; \quad k^{[i]}=\frac{(k+i) !}{k !} ; i=1,2, \ldots
$$

$P(\zeta=0)=y_{0}^{*}=x_{0}^{*} / x^{*}$ is the percentage of substance that is located in the first cell of the channel. Let this percentage be

$$
y_{0}^{*}=\frac{a}{a+k}
$$

The case described by Eq.(11) corresponds to the situation where the amount of substance in the first cell is proportional of the amount of substance in the entire channel (self-reproduction property of the substance). In this case Eq.(10) is reduced to the distribution:

$$
P(\zeta=i)=\frac{a}{a+k} \frac{(k-1)^{[i]}}{(a+k)^{[i]}} ; \quad k^{[i]}=\frac{(k+i) !}{k !} ; i=1,2, \ldots
$$

Let us denote $\rho=a$ and $k=l$. The distribution (13) is exactly the Waring distribution (probability distribution of non-negative integers named after Edward Waring - a Lucasian professor of Mathematics in Cambridge in the 18th century) [9], 16, [17]

$$
p_{l}=\rho \frac{\alpha_{(l)}}{(\rho+\alpha)_{(l+1)}} ; \alpha_{(l)}=\alpha(\alpha+1) \ldots(\alpha+l-1)
$$

Waring distribution may be written also as follows

$$
\begin{aligned}
& p_{0}=\rho \frac{\alpha_{(0)}}{(\rho+\alpha)_{(1)}}=\frac{\rho}{\alpha+\rho} \\
& p_{l}=\frac{\alpha+(l-1)}{\alpha+\rho+l} p_{l-1} .
\end{aligned}
$$

The mean $\mu$ (the expected value) of the Waring distribution is

$$
\mu=\frac{\alpha}{\rho-1} \text { if } \rho>1
$$


The variance of the Waring distribution is

$$
V=\frac{\alpha \rho(\alpha+\rho-1)}{(\rho-1)^{2}(\rho-2)} \text { if } \rho>2
$$

$\rho$ is called the tail parameter as it controls the tail of the Waring distribution. Waring distribution contains various distributions as particular cases. Let $i \rightarrow \infty$ Then the Waring distribution is reduced to

$$
p_{l} \approx \frac{1}{l^{(1+\rho)}} .
$$

which is the frequency form of the Zipf distribution [5]. If $\alpha \rightarrow 0$ the Waring distribution is reduced to the Yule-Simon distribution [24]

$$
p(\zeta=l \mid \zeta>0)=\rho B(\rho+1, l)
$$

where $B$ is the beta-function.

\subsection{Non-stationary regime of functioning of the channel}

In the nonstationary case $d x_{0} / d t \neq 0$. In this case the solution of the first equation of the system of equations (5) is

$$
x_{0}=b_{00} \exp \left[\left(\sigma-\alpha-\gamma_{0}\right) t\right]
$$

where $b_{00}$ is a constant of integration. $x_{i}$ must be obtained by solution of the corresponding Eqs.(15). The form of $x_{i}$ is

$$
x_{i}=\sum_{j=0}^{i} b_{i j} \exp \left[-\left(\alpha+\beta j+\gamma_{j}-\sigma_{j}\right) t\right]
$$

The solution of the system of equations (51) is (21) where $\sigma_{i}=0$, $i=1, \ldots$, :

$$
b_{i j}=\frac{\alpha+\beta(i-1)}{\gamma_{i}-\gamma_{j}+\beta(i-j)} b_{i-1, j} ; i=1, \ldots,
$$

and $b_{i i}$ are determined from the initial conditions in the cells of the channel. The asymptotic solution $(t \rightarrow \infty)$ is

$$
x_{i}^{a}=b_{i 0} \exp \left[\left(\sigma-\alpha-\gamma_{0}\right) t\right]
$$

This means that the asymptotic distribution $y_{i}^{a}=x_{i}^{a} / x^{a}$ is stationary

$$
y_{i}^{a}=\frac{b_{i 0}}{\sum_{j=0}^{\infty} b_{j 0}}
$$


regardless of the fact that the amount of substance in the two cells may increase or decrease exponentially. The explicit form of this distribution is

$$
y_{0}^{a}=\frac{1}{1+\sum_{i=1}^{\infty} \prod_{k=1}^{i} \frac{\alpha+\beta(k-1)}{\gamma_{k}-\gamma_{0}+\beta k}}, \quad y_{i}^{a}=\frac{\prod_{k=1}^{i} \frac{\alpha+\beta(k-1)}{\gamma_{k}-\gamma_{0}+\beta k}}{\sum_{i=0}^{\infty} \prod_{k=1}^{i} \frac{\alpha+\beta(k-1)}{\gamma_{k}-\gamma_{0}+\beta k}}, i=1, \ldots
$$

\section{The model of two substances}

Let us discuss now a model of moving of two immiscible substances through a channel containing infinite number of cells. The substances enter the channel through the entry cell and in general the following three processes are allowed: the substances may enter the cells one after the another and the substances may be used for some purposes in the corresponding cell. From the point of view of migration flows this model corresponds to migration of migrants with two different values of some characteristics (e.g. different religions).

Let us denote the amount of substance of the two types in the $i$-th cell of the channel as $x_{i}^{1}$ and $x_{i}^{2}$. The model equations for the movement of the two kings of substance are

$$
\begin{aligned}
& \frac{d x_{0}^{1}}{d t}=\sigma^{1} x_{0}^{1}-\alpha^{1} x_{0}^{1}-\gamma_{0}^{1} x_{0}^{1} ; \\
& \frac{d x_{i}^{1}}{d t}=\left[\alpha^{1}+\beta^{1}(i-1)\right] x_{i-1}^{1}-\left(\alpha^{1}+\beta^{1} i+\gamma_{i}^{1}\right) x_{i}^{1} ; \quad i=1,2, . \\
& \frac{d x_{0}^{2}}{d t}=\sigma^{2} x_{0}^{2}-\alpha^{2} x_{0}^{2}-\gamma_{0}^{2} x_{0}^{2} \\
& \frac{d x_{i}^{2}}{d t}=\left[\alpha^{2}+\beta^{2}(i-1)\right] x_{i-1}^{2}-\left(\alpha^{2}+\beta^{2} i+\gamma_{i}^{2}\right) x_{i}^{2} ; \quad i=1,2, .
\end{aligned}
$$

For the stationary regime of functioning of the channel the amount of the substances and the stationary distributions of the substances in for the two kinds of substances are

$$
\begin{aligned}
x_{i}^{1, *} & =\frac{\left[k^{1}+(i-1)\right] !}{\left(k^{1}-1\right) ! \prod_{j=1}^{i}\left(k^{1}+j+a_{j}^{1}\right)} x_{0}^{1, *} \\
y_{i}^{1, *} & =\frac{\left[k^{1}+(i-1)\right] !}{\left(k^{1}-1\right) ! \prod_{j=1}^{i}\left(k^{1}+j+a_{j}^{1}\right)} y_{0}^{1, *}
\end{aligned}
$$




$$
\begin{aligned}
& x_{i}^{2, *}=\frac{\left[k^{2}+(i-1)\right] !}{\left(k^{2}-1\right) ! \prod_{j=1}^{i}\left(k^{2}+j+a_{j}^{2}\right)} x_{0}^{2, *} \\
& y_{i}^{2, *}=\frac{\left[k^{2}+(i-1)\right] !}{\left(k^{2}-1\right) ! \prod_{j=1}^{i}\left(k^{2}+j+a_{j}^{2}\right)} y_{0}^{2, *}
\end{aligned}
$$

where $k^{1}=\alpha^{1} / \beta^{1} ; k^{2}=\alpha^{2} / \beta^{2} ; a_{j}^{1}=\gamma_{j}^{1} / \beta^{1} ; a_{j}^{2}=\gamma_{j}^{2} / \beta^{2} ; y_{i}^{1, *}=$ $x_{i}^{1, *} / x^{1, *} ; y_{i}^{2, *}=x_{i}^{2, *} / x^{2, *}$ and $x^{1, *}$ and $x^{2, *}$ are the total amounts of the two substances in all cells of the channel.

For the case of non-stationary regime of functioning of the channel the forms of the asymptotic distribution for the two kinds of substances are

$$
\begin{gathered}
y_{0}^{1, a}=\frac{1}{1+\sum_{i=1}^{\infty} \prod_{k=1}^{i} \frac{\alpha^{1}+\beta^{1}(k-1)}{\gamma_{k}^{1}-\gamma_{0}^{1}+\beta^{1} k}}, \quad y_{i}^{1, a}=\frac{\prod_{k=1}^{i} \frac{\alpha^{1}+\beta^{1}(k-1)}{\gamma_{k}^{1}-\gamma_{0}^{1}+\beta^{1} k}}{\sum_{i=0}^{\infty} \prod_{k=1}^{i} \frac{\alpha^{1}+\beta^{1}(k-1)}{\gamma_{k}^{1}-\gamma_{0}^{1}+\beta^{1} k}}, i=1, \ldots \\
y_{0}^{2, a}=\frac{1}{1+\sum_{i=1}^{\infty} \prod_{k=1}^{i} \frac{\alpha^{2}+\beta^{2}(k-1)}{\gamma_{k}^{2}-\gamma_{0}^{2}+\beta^{2} k}}, \quad y_{i}^{2, a}=\frac{\prod_{k=1}^{i} \frac{\alpha^{2}+\beta^{2}(k-1)}{\gamma_{k}^{2}-\gamma_{0}^{2}+\beta^{2} k}}{\sum_{i=0}^{\infty} \prod_{k=1}^{i} \frac{\alpha^{2}+\beta^{2}(k-1)}{\gamma_{k}^{2}-\gamma_{0}^{2}+\beta^{2} k}}, i=1, \ldots
\end{gathered}
$$

\section{Discussion}

We have mentioned above that the discussed model can be used for study of movement and distribution of migrants in a sequence of countries that form a migration channel. Migration channel of such kind was clearly visible in 2015 when a large influx of migrants in Europe was observed along a channel with Greece as the entry country. The parameters of the models discussed above can be interpreted as follows from the point of view of the application of the models to the migration channels. $\sigma$ can be considered as a "gate" parameter as it regulates the number of migrants that enter the channel. $\sigma$ is a parameter that is specific for the entry country of the channel. A small value of $\sigma$ can decrease significantly the number of migrants that enter the channel. Large value of $\sigma$ may lead to large migration flows. The value of the parameter $\sigma$ can be regulated by the authorities of the entry country and by some over-national authorities if such authorities exist and they are allowed to act on the territory of the entry country. Thus if the state structures of the entry country are weak 
because of some kind of crisis or as a consequences of other reasons then the value of the parameter $\sigma$ may be large. Thus large number of migrants may enter the channel and this will lead to large problems in the entry country and in all countries along the channel, especially to the countries that are close the entry country and are part of the migration channel.

Another kind of "gate" parameter is the parameter $\alpha$ that regulates the number of migrants that move from one country to the next country in the sequence of countries that form the migration channel. Large value of $\alpha$ means that the movement between the countries is large and the migrants easily cross the state borders. Small value of $\alpha$ means that the the crossing of the borders is more difficult. What was observed for the case of the migration flows in 2015 in Europe was that at some moments the borders have been practically open and for some time the value of the parameter $\alpha$ was large and almost the same for all countries that have been part of the channel. Such kind of situation is modelled by the models presented above.

The parameter $\beta$ accounts for the attractiveness of the countries of the channel that are distant from the entry country of the channel. The large values of this parameter lead to a tendency for leaving the countries around the beginning of the migration channel and attempts to settle in much more attractive countries along the channel. Parameter $\gamma_{i}$ accounts for the number of migrants that enter the $i$-th country of the channel but do not leave it. The reasons for this may be different, e.g. some migrants may obtain permission to stay in the country. Small values of the parameters $\gamma_{i}$ correspond to a large traffic of migrants through the countries of the channel. If in some country the value of the corresponding parameter $\gamma$ is large then significant number of migrants may stay in this country and the number of the migrant moving further through the channel may decrease.

For the case of two kinds of migrants the number of parameters increase which leads to increasing number of possible situations. For an example the entry country may prefer one of the kinds of migrants and then the values of the "gate" parameters $g^{1}$ and $g^{2}$ may have quite different values. The countries along the channel may impose different level of difficulty of crossing the borders which may lead in differences in the values of the parameters $\alpha^{1}$ and $\alpha^{2}$. The countries along the channel may have different level of attractiveness which will lead to different values of the parameters $\beta^{1}$ and $\beta^{2}$. Finally different countries may have different preferences about the numbers and about the kind of migrants they allow to stay in the country. This will lead to different values of the parameters $\gamma_{i}^{1}$ and $\gamma_{i}^{2}$.

Let us discuss the above remarks in more detail by means of the 
obtained mathematical results. Let us first consider the case of one kind of migrants and stationary regime of functioning of the channel. From Eq.(10) we obtain

$$
\frac{y_{i}^{*}}{y_{i+1}^{*}}=1+\frac{\beta+\gamma_{i+1}}{\alpha+\beta i}
$$

Eq.(34) shows us that for the case of infinite channel the number of migrants has to decrease with increasing value of $i$, i.e., in the countries that are away from the entry country of the channel the number of migrants in any country is smaller than the number of migrants in the previous country of the channel. Then for the case of infinite channel the effect of concentration of migrants in countries that are far from the entry country is not observed even if these countries are very attractive to the migrants. Let us note here that the models considered above can be extended to the case of a channel containing finite number of cells (finite number of countries for the vase of migrant flows). Then a new effect will be observed: concentration of migrants in the last cell of the channel (the final destination country). If the attractiveness of the final destination country is large then the concentration of the migrants will be observed in the entry country of the channel and in the final destination country. Such possible developments of the situation in the migration channels will be discussed elsewhere.

Eq.(34) shows additional details about the influence of the parameters of the channel on the migrant flow. The increase of $\alpha$ leads to decreasing of the ratio $y_{i}^{*} / y_{i+1}^{*}$. This means that the larger value of the gate parameter $\alpha$ leads to a smoothing of the distribution of the number of migrants along the countries of the channel. Thus if the countries that are far from the entry country of the channel want to decrease the number of migrants on its own territory the have to take measures to decrease the value of the parameter $\alpha$. The increasing of the value of the parameter $\beta$ has more complicated influence on the ratio $y_{i}^{*} / y_{i+1}^{*}$ but in principle the effect is the same as the increasing of the value of parameter $\alpha$. If the countries that are attractive for migrants have a policy to decrease their attractiveness then smaller number of migrants will reach their territory. If such countries have "open doors" politics towards migrants then larger number of migrants will leave the countries around the entry country of the channel and will move towards the attractive countries. In combination with large value of $\sigma$ this may lead to floods of migrants in the attractive countries. But this will lead also to even larger flood of migrants in the countries that are around the entry country of the channel. This was observed indeed for the case of the massive migration in Europe in 2015. Finally the increasing value of the "leakage" parameter $\gamma_{i+1}$ 
will lead to decreasing of the ratio $y_{i}^{*} / y_{i+1}^{*}$. The reason for this is obvious: when more migrants change their migrant status (e.g. obtain permission to stay) in the the $i+1$-th country then the number of migrants without status will decrease and this will lead to decreasing ratio $y_{i}^{*} / y_{i+1}^{*}$.

Let us now discuss further the case of two kinds of migrants for the stationary regime of the functioning of the channel. In this case the ratio of the numbers of migrants in a cell of the channel is

$$
\frac{x_{i}^{1, *}}{x_{i}^{2, *}}=\frac{x_{0}^{1, *}}{x_{0}^{2, *}} \frac{\left[k^{1}+(i-1)\right] !}{\left[k^{2}+(i-1)\right] !} \frac{\left(k^{2}-1\right) !}{\left(k^{1}-1\right) !} \frac{\prod_{j=1}^{i}\left(k^{2}+j+a_{j}^{2}\right)}{\prod_{j=1}^{i}\left(k^{1}+j+a_{j}^{1}\right)}
$$

Eq.(35) leads to the following conclusions. First of all the mix of the migrants in any country of the channel depends on the ratio $x_{0}^{1, *} / x_{0}^{2, * *}$ at the entry country of the channel. Thus the politics in the entry country of the channel towards the different categories of migrants is extremely important for the distribution of migrants in the entire channel. If there are migrants with unfavorable characteristics from the point of view of countries from the the channel then one of most effective ways to reduce the number of such migrants is to reduce their possibility to enter the channel. The influence of the other parameters of the channel is more complicated. Large values of $\gamma_{i}^{2}$ with respect to the values of $\gamma_{i}^{1}$ (this corresponds, e.g., for much larger acceptance of the migrants of class 2 in comparison to the migrants of class 1 in all countries of the channel up to the $i$-th country of the channel) when the other parameters of the channel are equal for the two classes of migrants lead to larger value of the ratio $x_{0}^{1, *} / x_{0}^{2, *}$. This means that the mix of migrants without status in the $i$-th country of the channel depends on the politics of previous countries of the channel towards different categories of migrants. Finally the ratio of the migrants depends on the parameters $k^{1}$ and $k^{2}$ that are ratios $\alpha^{1} / \beta^{1}$ and $\alpha^{2} / \beta^{2}$, i.e. the ratios between corresponding possibility for mobility of the class of migrants (the parameters $\alpha^{1}$ and $\alpha^{2}$ ) and the attractiveness of the countries far away from the entry country of the channel (the parameters $\beta^{1}$ and $\beta^{2}$ ). This dependence is the most complicated one. If the ratios $k^{1}$ and $k^{2}$ are the same then the influence of $k^{1}$ and $k^{2}$ adds nothing to the influence of parameters $a^{1}$ and $a^{2}$ on the mix of migrants in the countries of the channel. In order to obtain more information about the influence of $k^{1,2}$ (respectively about the influence of the parameters $\alpha^{1,2}$ and $\beta^{1,2}$ we can use the recurrence relationships for the numbers of migrants in the $i$-th country of the 
channel. These relationships may be written as

$$
x_{i}^{1, *}=\frac{1}{1+\frac{\beta^{1}+\gamma_{i}^{1}}{\alpha^{1}+\beta^{1}(i-1)}} x_{i-1}^{1, *}, \quad x_{i}^{2, *}=\frac{1}{1+\frac{\beta^{2}+\gamma_{i}^{2}}{\alpha^{2}+\beta^{2}(i-1)}} x_{i-1}^{2, *}
$$

Then the vary large values of the parameter $\beta$ increase the traffic of migrants through the channel and the decrease of the number of migrants in the $i$ in comparison to the number of migrants in $i-1$-th country will be approximately $1-1 / i$ as a proportion. The influence of the very large values of the parameters $\alpha$ is larger. When the value of the parameter $\alpha$ is much large that the values of the corresponding parameters $\beta$ and $\gamma_{i}$ then the number of migrants in the $i$-th country of the channel will be approximately equal to the number of migrants in the $i$-th country of the channel. Thus the decreasing permeability of the borders between the neighbouring states from the migration channel is more effective that decreasing of the attractiveness of the countries far from the entry country of the channel.

Let us mention finally several methodological renarks. The model presented above was a linear one. One possible extention is to take into account nonlinear effects. Then the model system will contain nonlinear ODEs or nonlinear PDEs. Such equations may be soved analytically in some particular cases [6] - 8], [33] - [36], [41, 42], but in the most cases one has to solve them numerically. The corresponding statistical distrubutions must be obtained numerically too.

\section{References}

[1] Armitage R.. Population projections for English local authority areas. Population Trends 43 (Spring), 31-40 (1986).

[2] Borjas G. J. Economic theory and international migration. International Migration Review 23, 457 - 485 (1989).

[3] Bracken I., Bates J. J. Analysis of gross migration profiles in England and Wales: some developments in classification. Environment and Planning A 15, 343-355 (1983).

[4] Champion A. G., Bramley G., Fotheringham A. S., Macgill J., Rees P. H. A migration modelling system to support government decision-making. In Stillwell J., Geertman S. (Eds.) Planning support systems in practice. p.p. 257 - 278 Springer Verlag, Berlin (2002).

[5] Chen W.-C.. On the weak form of the Zipf's law. Journal of Applied Probability 17, 611 - 622 (1980). 
[6] Dimitrova Z. I., Vitanov N. K. Influence of adaptation on the nonlinear dynamics of a system of competing populations. Physics Letters A 272, 368-380 (2000).

[7] Dimitrova Z. I., Vitanov, N. K. Adaptation and its impact on the dynamics of a system of three competing populations. Physica A: Statistical Mechanics and its Applications 300 , 91-115 (2001).

[8] Dimitrova, Z. I., Vitanov, N. K. Dynamical consequences of adaptation of the growth rates in a system of three competing populations. Journal of Physics A: Mathematical and General 34, 7459 - 7473 (2001).

[9] Diodato V. Dictionary of bibliometrics. Haworth Press, Binghampton, NY (1994).

[10] Ethier W. J. International trade and labor migration. The American Economic Review 75, 691 - 707 (1985).

[11] Fawcet, J. T.. Networks, linkages, and migration systems. International Migration Review 23, 671 - 680 (1989).

[12] Greenwood M. J. Modeling migration. In Kemp-Leonard K. (Ed.) Encylopedia of social measurement, vol. 2, p.p. 725 - 734 Elsevier, Amsterdam (2005).

[13] Gurak D. T., Caces F.. Migration networks and the shaping of migration systems. In: Kitz M.M., Lim L. L., Zlotnik H. (Eds.) International migration systems: A global approach.p.p. 150-176 Clarendon Press, Oxford, (1992).

[14] Harris J. R., Todaro M. P. Migration, unemployment and development: A two-sector analysis. The American Economic Review 60, 126 - 142 (1970).

[15] Hotelling H. A mathematical theory of migration. Environment and Planning 10, 1223 - 1239 (1978).

[16] Irwin J. O. The place of mathematics in medical and biological sciences. Journal of the Royal Statistical Society 126, 1 - 44 (1963).

[17] Irwin J. O. The generalized Waring distribution applied to accident theory. Journal of the Royal Statistical Society 131, 205 225 (1968).

[18] Ledent J. Multistate life table: movement versus transition perspectives. Environment and Planning A 12, 533 - 562 (1980).

[19] Lee E. S. A theory of migration. Demography 3, 47 - 57 (1966).

[20] Massey D. S., Arango J., Hugo G., Kouaougi A., Pellegrino A., Edward Taylor J.. Theories of international migration: A review 
and appraisal. Population and Development Review 19, 431 466 (1993).

[21] Puu T. Hotelling's migration model revisited. Environment and Planning 23, 1209 - 1216 (1991).

[22] Puu T. A simplified model of spatiotemporal population dynamics. Environment and Planning 17, 1263 - 1269 (1985).

[23] Schubert A., Glänzel W. A dynamic look at a class of skew distributions. A model with scientometric application. Scientometrics 6, 149 - 167 (1984).

[24] Simon H. A.. On a class of skew distribution functions. Biometrica 42, $425-440$ (1955).

[25] Simon J. H. The economic consequences of migration. The University of Michnigan Press, Ann Arbor, MI (1999).

[26] Skeldon R. Migration and development: A global perspective. Routledge, London (1992).

[27] Vitanov N. K. Science dynamics and research production: Indicators, indexes, statistical laws and mathematical models. Springer International (2016).

[28] Vitanov N.K., Vitanov K. N. Population dynamics in presence of state dependent fluctuations. Computers \& Mathematics with Applications 68, 962 - 971. (2013).

[29] Vitanov N. K., Vitanov K. N. Box model of migration channels. Mathematical Social Sciences 80, 108 - 114 (2016).

[30] Vitanov N. K., Jordanov I. P., Dimitrova Z. I.. On nonlinear dynamics of interacting populations: Coupled kink waves in a system of two populations. Communications in Nonlinear Science and Numerical Simulation 14, 2379 - 2388 (2009)

[31] Vitanov N. K., Jordanov I. P., Dimitrova Z. I.. On nonlinear population waves. Applied Mathematics and Computation 215, 2950 - 2964 (2009).

[32] Vitanov N. K., Dimitrova Z. I., M. Ausloos. Verhulst-LotkaVolterra model of ideological struggle. Physica A 389, 4970 4980 (2010).

[33] Vitanov N. K. Application of simplest equations of Bernoulli and Riccati kind for obtaining exact traveling-wave solutions for a class of PDEs with polynomial nonlinearity. Communications in Nonlinear Science and Numerical Simulation 15, 2050-2060 (2010) 
[34] Vitanov N. K., Dimitrova, Z. I. Application of the method of simplest equation for obtaining exact traveling-wave solutions for two classes of model PDEs from ecology and population dynamics. Communications in Nonlinear Science and Numerical Simulation 15, 2836-2845 (2010)

[35] Vitanov N.K., Dimitrova Z.I., Kantz, H. Modified method of simplest equation and its application to nonlinear PDEs. Applied Mathematics and Computation 216, 2587-2595 (2010).

[36] Vitanov N. K. Modified method of simplest equation: powerful tool for obtaining exact and approximate traveling-wave solutions of nonlinear PDEs. Communications in Nonlinear Science and Numerical Simulation 16, 1176-1185 (2011)

[37] Vitanov N. K., Ausloos M., Rotundo G. Discrete model of ideological struggle accounting for migration. Advances in Complex Systems 15, Supplement 1, Article number 1250049 (2012).

[38] Vitanov N. K., Ausloos M. Knowledge epidemics and population dynamics models for describing idea diffusion. p.p. 65-129 in A. Scharnhorst, K Boerner, P. van den Besselaar (Eds.) Models of Science Dynamics, Springer, Berin (2012).

[39] Vitanov N.K., Dimitrova Z. I. On waves and distributions in population dynamics. BIOMATH 1, Art. No. 1209253 (2012).

[40] Vitanov N. K., Dimitrova Z. I., Vitanov K. N. Traveling waves and statistical distributions connected to systems of interacting populations. Computers \& Mathematics with Applications 66, 1666 - 1684 (2013).

[41] Vitanov N. K., Dimitrova Z. I. Solitary wave solutions for nonlinear partial differential equations that contain monomials of odd and even grades with respect to participating derivatives. Applied Mathematics and Computation 247, 213 - 217 (2014)

[42] Vitanov N.K., Dimitrova Z. I., Vitanov K. N.Modified method of simplest equation for obtaining exact analytical solutions of nonlinear partial differential equations: further development of the methodology with applications. Applied Mathematics and Computation 269, 363-378 (2015).

[43] Vitanov N. K., Ausloos M. Test of two hypotheses explaining the size of populations in a system of cities. Journal of Applied Statistics 42, 2686-2693 (2015).

[44] Weidlich W., Haag G. (Eds.). Interregional migration. Dynamic theory and comparative analysis. Springer, Berlin (1988). 
[45] Willekens F. J. Probability models of migration: Complete and incomplete data. SA Journal of Demography 7, 31 - 43 (1999).

[46] Willekens F. Models of migration observations and judgement. In Raymer J, Willekens F. (Eds.) International migration in Europe: Data, models and estimates. p.p. 117-147, Wiley, New York (2008). 